\title{
THE CLASS OF RECURSIVE FUNCTIONS
}

\author{
J. R. SHOENFIELD
}

In this note, we find the position of the predicate " $\alpha$ is recursive" in the Kleene arithmetical hierarchy. ${ }^{1}$ The proof involves a use of topology; the key step is the application of Baire's category theorem to a suitable function space.

We use the notation of [1]. In addition, we write $(U x)$ for the quantifier "there exist infinitely many $x$."

We designate the set of natural numbers by $N$ and the class of mappings of $N$ into $N$ by $N^{N}$. We consider $N$ as a topological space with the discrete topology, and $N^{N}$ as a topological space with the product topology. We review some known facts about $N^{N}$.

I. Provided with a suitable metric, $N^{N}$ is a complete metric space. (For it is the product of a countable number of discrete spaces.)

II. The nonrecursive functions are dense in $N^{N}$. (For a non recursive function remains nonrecursive if its values at a finite number of arguments are changed.)

III. If $R(\alpha)$ is a recursive predicate, then $\hat{\alpha}(R(\alpha))$ is open and closed. (Since the complement of $\hat{\alpha}(R(\alpha))$ is $\hat{\alpha}(\bar{R}(\alpha))$, it is sufficient to prove $\hat{\alpha}(R(\alpha))$ open. This follows from the fact that if $R(\alpha)$, then $R(\beta)$ for any function $\beta$ agreeing with $\alpha$ on a certain finite set of arguments.)

Lemma. ${ }^{2}$ For every predicate $R(\alpha, x, y, z)$ there is a predicate $S(\alpha, x, y)$ recursive in $R$ such that

$$
(x)(E y)(z) R(\alpha, x, y, z) \equiv(U x)(y) S(\alpha, x, y)
$$

for all $\alpha$.

Proof. Define

$$
\begin{aligned}
& C(x, \alpha)=\hat{y}(E z) \bar{R}(\alpha, x, y, z), \\
& D(x, \alpha)=\hat{w}(y)_{y<w}(y \in C(x, \alpha)), \\
& E(v, \alpha)=\bigcup_{x<v} D(x, \alpha) .
\end{aligned}
$$

Then

Presented to the Society, January 30, 1958; received by the editors April 3, 1958.

1 This problem was suggested to the author by John Addison.

2 This lemma was suggested to the author by a proof due to Hartley Rogers. 


$$
\begin{aligned}
E(v, \alpha) \text { is finite } & \equiv(x)_{x<v} D(x, \alpha) \text { is finite } \\
& \equiv(x)_{x<v}(E y)(y \notin C(x, \alpha)) \\
& \equiv(x)_{x<v}(E y)(z) R(\alpha, x, y, z) .
\end{aligned}
$$

Hence

$$
\begin{aligned}
(x)(E y)(z) R(\alpha, x, y, z) & \equiv(U v)(x)_{x<v}(E y)(z) R(\alpha, x, y, z) \\
& \equiv(U v) E(v, \alpha) \text { is finite. }
\end{aligned}
$$

Now $E(v, \alpha)$ is clearly recursively enumerable in $R$ uniformly in $v$ and $\alpha$. Hence by the enumeration theorem, there is an $e$ such that

$$
z \in E(v, \alpha) \equiv(E w) T_{2}^{R, \alpha}(e, v, z, w) .
$$

We recall that for each $v, z$ there is at most one $w$ such that $T_{2}^{R, \alpha}(e, v, z, w)$. It readily follows that

$$
\begin{aligned}
(U v) E(v, \alpha) \text { is finite } & \equiv(U x)(y)\left(T_{2}^{R, \alpha}\left(e,(x)_{0},(x)_{1},(x)_{2}\right) \& \ln (x)\right. \\
& \left.\leqq 3 \&\left((y)_{0}>(x)_{1} \rightarrow \bar{T}_{2}^{R, \alpha}\left(e,(x)_{0},(y)_{0},(y)_{1}\right)\right)\right) .
\end{aligned}
$$

Combining with (1), we get the lemma.

Theorem. The predicate " $\alpha$ is recursive" can be written in the form $(E x)(y)(E z) R(\alpha, x, y, z)$ with $R$ recursive but cannot be written in the form $(x)(E y)(z) R(\alpha, x, y, z)$ with $R$ recursive.

Proof. The first part is immediate, since

$$
\alpha \text { is recursive } \equiv(E x)(y)(E z)\left(T_{1}(x, y, z) \& U(z)=\alpha(y)\right) .
$$

Now suppose " $\alpha$ is recursive" could be written in the form $(x)(E y)(z)$ $R(\alpha, x, y, z)$ with $R$ recursive. Then by the lemma and the enumeration theorem we would have

$$
\alpha \text { is recursive } \equiv(U x)(y) \bar{T}_{1}^{1}(\bar{\alpha}(y), e, x)
$$

for a suitable $e$. We shall show that this leads to a contradiction.

Let $A(\alpha)=\hat{x}(y) \bar{T}_{1}^{1}(\bar{\alpha}(y), e, x)$. If $\alpha$ is nonrecursive, then $A(\alpha)$ is finite by (2). Since there are only countably many recursive functions and only countably many finite sets of natural numbers, there are only countably many different sets $A(\alpha)$. Hence the sets $\hat{\alpha}(A(\alpha)=B)$ with $B$ a set of natural numbers form a countable covering of $N^{N}$. By I above and Baire's category theorem, it follows that there is a set $B$ such that, setting $\mathfrak{C}=\hat{\alpha}(A(\alpha)=B), \overline{\mathrm{e}}$ has an interior point.

Since the nonrecursive functions are dense, there is a nonrecursive function $\beta$ in $\overline{\mathfrak{e}}$. Now for any $x$, 


$$
\hat{\alpha}(x \in A(\alpha))=\alpha(y) \bar{T}_{1}^{1}(\bar{\alpha}(y), e, x)=\bigcap_{y} \alpha \bar{T}_{1}^{1}(\bar{\alpha}(y), e, x)
$$

is closed by III above. Thus

$$
x \in B \rightarrow \mathfrak{e} \subseteq \hat{\alpha}(x \in A(\alpha)) \rightarrow \overline{\mathcal{C}} \equiv \hat{\alpha}(x \in A(\alpha)) \rightarrow x \in A(\beta) .
$$

Thus $B \subseteq A(\beta)$. Since $A(\beta)$ is finite, $B$ is finite.

Let $k=\max B$. Since $\overline{\mathfrak{C}}$ has an interior point, there is a sequence number $s>0$ such that

$$
(E y)(\bar{\alpha}(y)=s) \rightarrow \alpha \in \overline{\mathrm{C}} .
$$

Set

$$
\begin{aligned}
\gamma(0) & =s, \\
\gamma(n+1) & =\mu w\left(\operatorname{Ext}(w, \gamma(n)) \& w>\gamma(n) \& T_{1}^{1}(w, e, k+n+1)\right)
\end{aligned}
$$

(where $\operatorname{Ext}(w, z)$ means that $w$ and $z$ are sequence numbers and that $(w)_{i}=(z)_{i}$ for all $\left.i<\operatorname{lh}(z)\right)$. We show that $\gamma$ is well defined. Assume $\gamma(n)$ is defined. Then $\gamma(n)$ is a sequence number; so $\gamma(n)=\bar{\delta}(y)$ for some $\delta$ and $y$. By (3), $\delta \in \overline{\mathcal{C}}$. Hence there is a $\sigma$ in $\mathcal{C}$ such that $\bar{\sigma}(y)$ $=\bar{\delta}(y)=\gamma(n)$. Now $k+n+1 \notin B=A(\sigma)$. Hence for some $u$,

$$
T_{1}^{1}(\bar{\sigma}(u), e, k+n+1) ;
$$

and we may suppose $u>y$. If $w=\bar{\sigma}(u)$, then $\operatorname{Ext}(w, \gamma(n)) \& w>\gamma(n)$ $\& T_{1}^{1}(w, e, k+n+1)$. Hence $\gamma(n+1)$ is defined.

Clearly $\gamma$ is recursive. Let $\alpha(n)=(\gamma(n))_{n}-1$; then $\alpha$ is recursive. Now for each $n, \gamma(n+1)=\bar{\alpha}(y)$ for some $y$; so $T_{1}^{1}(\bar{\alpha}(y), e, k+n+1)$ and hence $k+n+1 \notin A(\alpha)$. It follows that $A(\alpha)$ is finite. But this contradicts (2).

\section{REFERENCES}

1. S. C. Kleene, Hierarchies of number-theoretic predicates, Bull. Amer. Math. Soc. vol. 61 (1955) pp. 193-213.

DUKE UNIVERSITY 